\title{
Ischemic Mitral Regurgitation
}

\author{
Hashem Aliter ${ }^{1}$ \\ ${ }^{1}$ Dalhousie University
}

May 7, 2020

\begin{abstract}
Ischemic mitral regurgitation (IMR) is one of the most challenging complications of acute myocardial infarction (AMI) and chronic coronary artery disease (CAD). The presence of IMR, irrespective of severity significantly have a negative impact on the short and long-term outcomes of patients with AMI and CAD. Nearly 25\% of patients with AMI without congestive heart failure (CHF) develop IMR and this number approaches $50 \%$ when $\mathrm{CHF}$ is present.
\end{abstract}

\section{Ischemic Mitral Regurgitation}

Hashem Aliter ${ }^{*}$

${ }^{1}$ Division of Cardiac surgery, Dalhousie University, Halifax, Canada

${ }^{*}$ Corresponding author: Hashem Aliter, Division of Cardiac surgery, Dalhousie University, Halifax, Canada. Tel: +1-9024127979; Fax: +1-9024733808; Email:alitermd@gmail.com

Abstract

Ischemic mitral regurgitation (IMR) is one of the most challenging complications of acute myocardial infarction (AMI) and chronic coronary artery disease (CAD). The presence of IMR, irrespective of severity significantly have a negative impact on the short and long-term outcomes of patients with AMI and CAD. Nearly $25 \%$ of patients with AMI without congestive heart failure (CHF) develop IMR and this number approaches $50 \%$ when $\mathrm{CHF}$ is present

Keywords: coronary artery disease, mitral regurgitation, surgery, transcatheter.

\section{Introduction}

Ischemic mitral regurgitation (IMR) is one of the most challenging complications of acute myocardial infarction (AMI) and chronic coronary artery disease (CAD). The presence of IMR, irrespective of severity significantly have a negative impact on the short and long-term outcomes of patients with AMI and CAD. Nearly $25 \%$ of patients with AMI without congestive heart failure (CHF) develop IMR and this number approaches $50 \%$ when CHF is present. IMR occurs in approximately $20-25 \%$ of patients followed up after myocardial infarction $(\mathrm{MI})^{[1-3]}$ and in $50 \%$ of those with post-infarct congestive heart failure $(\mathrm{CHF})^{[4]}$. In patients with CHF, even a mild degree of mitral regurgitation (MR) adversely affects survival. Increasing MR severity is associated with a progressively worse 5 -year survival rate ${ }^{[1,4]}$.

This review is a concise overview of IMR and we will attempt to focus on the fundamentals of IMR and potential treatments for this challenging disease state.

\section{Definition}

Dr. Carpentier has defined the triad of MR by focusing on the etiology, lesion and dysfunction, as such IMR can have a specific definition. The etiology of IMR is due to coronary artery disease (CAD) and 
virtually is always associated with AMI. The primary lesion in IMR is leaflet tethering resulting from left ventricle (LV) remodeling secondary to an acute ischemic insult. Multiple studies have shown that the inferior/inferoposterior MI is more commonly associated with $\mathrm{IMR}^{[5]}$. The dysfunction of IMR rises from posteriormedial and apical displacement of the papillary muscle (PM), which leads to a measurable "tethering distance" and ultimately results in apical tenting and restriction of the free margin of the leaflets and poor leaflet coaptation ${ }^{[6]}$. Tethering of secondary chordae can lead to a "seagull" deformation of the anterior leaflet, which has been targeted in certain repair series. Ischemic heart disease (IHD) results in LV and mitral annular remodeling ${ }^{[8]}$. As a consequence, LV remodeling can lead to changes in the geometry of the mitral valve apparatus leading to $\mathrm{MR}^{[9]}$.

It is noteworthy that IMR and FMR fundamentally have different pathophysiology, albeit IMR and FMR have been frequently used interchangeably in the literature. IMR is associated with LV remodeling, yet the degree of LV chamber remodeling differs due to the location of the AMI. Echocardiographic studies in patients with dilated non-ischemic cardiomyopathy confirm enlarged LV diameter and sphericity to be the major predictors of $\mathrm{MR}$ in addition to the tenting or apical tethering of the chordae tendineae and valve leaflets. However, IMR, especially in the inferior or infero-posterior AMI, is not as much dependent on global LV dilatation but rather on a more localized LV insult. Generally, IMR is more likely after inferior/inferoposterior AMI rather that anterior AMI. IMR associated with anterior infarction is usually accompanied by greater LV and mitral annular dilatation in the septal-lateral (S-L) (also known as "antero-posterior") axis, more impressive annular flattening, and lower ejection fraction (EF) relative to inferior MI. It appears that anterior infarction does not sufficiently affect critical annular-subvalvular spatial relationships that are perturbed by a smaller posterolateral infarct. As such, IMR in the presence of anterior AMI develops due to chronic remodeling and progressive LV dysfunction, which results in development of IMR. Thus, IMR associated with anterior MI may be more similar to the clinical spectrum to FMR characterized by severely depressed LV function, global LV dilatation, and annular enlargement. On the other hand, an inferior MI is associated with less global LV dilatation and systolic dysfunction but more substantial perturbation of the mitral subvalvular $\operatorname{apparatus}^{[10]}$.

\section{Prevalence and Risk Factors}

The true prevalence of IMR is unknown due to a number of factors. The heterogeneity of MR patients presented in different studies makes the assessment of the prevalence more challenging. In addition, the impact of the imaging modality used to identify MR or timing of imaging at the time of the diagnosis make the accurate assessment of MR less desirable [11]. As note earlier, IMR occurs in $25 \%$ of patients presenting with AMI without CHF and in $50 \%$ of patients with post-infarct CHF [1-4]. It is estimated that up to $20 \%$ of patients undergoing cardiac catheterization for symptomatic CAD [12] and nearly up to $28 \%$ of patients undergoing coronary artery bypass graft (CABG) [13] may have IMR.

The risk factors for developing IMR are; inferior AMI (38\%) versus anterior AMI (10\%) at echocardiographic follow-up after 2 years $[14,15]$, advanced age, female gender, prior acute MI, large infarct size, recurrent myocardial ischemia, multi-vessel CAD and CHF on admission [16].

\section{Impact of IMR on Prognosis}

Similar to the challenges of estimating the true prevalence of IMR, the prognosis of IMR and CAD is also heterogeneous in many studies. It is common in studies that patients with MR and CAD may have been considered as IMR, however, some patients may have MR yet the etiology of MR is not necessarily IMR. Nevertheless, the retrospective analyses of single center studies demonstrate that presence of MR always portends poor prognosis. Hickey et. al used the Duke Clinical Research Institute in 11,748 patients. The investigators demonstrated that that 1-year mortality for severe IMR was $40 \%$, for moderate IMR $17 \%$, for mild IMR $10 \%$ and for patients without IMR $6 \%$ [12].

A case-controlled study from Mayo Clinic demonstrated that patients with IMR detected in the chronic phase (more than 16 days) after Q-wave myocardial infarction have lower 5-year survival than patients without $\operatorname{MR}(38.5 \%$ vs $61.6 \%, \mathrm{p}<0.001)[2]$. 
The SAVE (Survival and Ventricular Enlargement) study demonstrated that mild IMR increases the risk of cardiovascular mortality, even in patients without CHF [3]. Patients

with IMR had a higher incidence of cardiovascular mortality $(29 \%$ vs $12 \%, \mathrm{p}<0.001)$ and CHF (24\% vs $16 \%, \mathrm{p}<0.001)$ than patients without IMR at a mean of 3.5 years after AMI.

\section{Pathophysiology and Mechanism of Regurgitation in IMR}

Although it is tempting to consider PM dysfunction or displacement, i.e. lack of contraction, as the main mechanism of regurgitation in IMR patients, animal and clinical studies have shown otherwise. In an elegant study by Kaul et. al, the investigators proved that it's not the PM dysfunction, but MR occurs during ischemia only when global LV function is affected. This mechanism applies even when thickening of the PM and the immediately adjacent LV remains intact. MR in this situation is related to incomplete mitral leaflet closure, i.e. the less the leaflet closure the more the MR [17]. Furthermore, Aguero et. al devised a left atrial infarction swine model. The investigators showed that left atrial infarction alone leads to significant left atrial enlargement and mitral annular dilatation that may result in IMR [18].

Multiple animal and clinical studies have demonstrated that the mitral leaflet area grow due to MR [1921]. Mitral valve leaflets are larger in IMR patients but fibrotic processes set in counterproductively. The endothelial-to-mesenchymal transition, an embryonic process is upregulated in these patients that leads to thickening of the leaflets of the mitral valve [22]. This study shows that despite increase in the absolute value of the mitral valve area, the profibrotic changes of the cell signaling pathways will lead to maladaptation of the coaptation of the leaflets.

Kalra et. al, in a cardiac MRI study of patients with moderate to severe IMR compared to mild MR patients demonstrated that the disposition of the PM was one of the main drivers of the regurgitation in IMR patients. In normal heart anatomy, the PMs are located below and slightly posterior to the commissures of the mitral valve leaflets. The physiologic function of the posterior PM is to move medially towards the septum and the anterior PM will follow the posterior PM and the distance between the two shortens. They measured the distance between the two PMs, defined as inter PM distance (IPMD). In this study, they noticed that the shortening of the IPMD was significantly less in IMR patients compared to controls. A cut-off point of $20 \mathrm{~mm}$ seems to increase the risk of $>$ moderate MR in patients with IMR. Moreover, they also showed that the reduced torsion of the posterior PM leads to increased MR. These findings elaborate the common feature of asymmetry of the line of coaptation of IMR at the level of P3 with severely posteriorly displaced P3 segment of the mitral valve leaflet, leading to severe MR and posteriorly directed jet.

The increase in preload caused by CIMR after MI is not accompanied by a parallel increase in contractility [24]. The chronic volume overload in a ventricle that has

decreased compliance causes an increase in wall stress and left atrial (LA), ventricular end-diastolic and wedge pressures. The LA and ventricle enlarge, resulting in pulmonary hypertension and congestion, leading ultimately to heart failure and death $[25,26]$. Ventricular dilatation increases tethering, which worsens MR severity, creating a cycle whereby MR begets MR in a self-perpetuating manner.

\section{Decision Making and Treatment:}

Medical treatment:

There are only a few studies that focus on the impact of medical treatment on CIMR. Medical treatment of CIMR may lead to a reduction in MR severity, and/or it may lead to

attenuation or reversion of post-MI LV remodeling. Several studies suggest that angiotensin-converting enzyme inhibitors, nitrates and diuretics can lead to a partial shorter long-term reduction in MR by increasing the transmitral valve pressure gradient through either afterload or preload reduction [27 - 29]. In addition, inotropic vasopressors, such as dobutamine, can decrease CIMR [30]. As shown by the SAVE (Survival And Ventricular Enlargement) and SOLVD (Studies of Left Ventricular Dysfunction) studies, ACE inhibition can attenuate [31], arrest [32] or reverse [33] post-MI LV remodeling. In addition, the CAPRICOR (Carvedilol 
PostInfarct Survival Controlled Evaluation) and CARMEN (Carvedilol and ACE Inhibitor Remodeling Mild Heart Failure Evaluation Trial) studies showed that the combination of ACE inhibition and b-blockade inhibits [34] or synergistically reverses LV negative remodeling. There are, however, no data from large trials that show a decrease in the incidence of CIMR after attenuation or reversal of LV remodeling with ACE inhibition and b-blockade [36]. Despite the use of these drugs CIMR remains common. Cardiac resynchronization therapy (CRT) significantly and immediately reduces functional MR and CIMR due to

improved coordinated timing of the PM insertion sites [36] and increased closing force. Long-term CRT (up to 12 months) results in progressive structural and functional LV reverse remodeling, improved LV systolic and diastolic function and decreased MR severity in patients with moderate-to-severe heart failure and dyssynchronous ventricular contraction [37]. This effect is also evident during exercise, preventing the increase of MR during exercise [38]. However, approximately 30\% of CHF patients treated with CRT do not respond to treatment [39]. Independent predictors of lack of response to CRT are ischemic heart disease, severe MR and LV end-diastolic dimension $75 \mathrm{~mm}$ [40]. This indicates that patients with CIMR are less likely to benefit from CRT, especially in advanced stages of LV dilatation and tenting [37].

Surgical treatment:

There are multiple single center studies published to tackle the treatment of IMR. Virtually ball of these studies are not randomized and there are numerous confounding factors for each study. Until recently, there was few randomized controlled trials (RCT) focusing on the surgical management of the IMR. Over the last decade, we have been able to randomize patients to mitral valve surgery and coronary artery bypass grafting (CABG) and $\mathrm{CABG}$ alone, as well as $\mathrm{CABG}+$ mitral valve repair vs. $\mathrm{CABG}+$ mitral valve replacement. The results of the RCT studies are summarized in Table - 1 [41-46].

When the mitral regurgitation is severe, debate has focused on the choice between MVR or chordal-preserving mitral valve replacement as recently addressed by Goldstein et. al who randomized 251 patients with chronic IMR to undergo either mitral-valve repair or chordal sparing replacement with complete preservation of the subvalvular apparatus [46]. At two years follow up, authors conclude that there was no difference with respect to LV reverse remodeling or survival but the rate of recurrence of moderate or severe mitral regurgitation was more than 15 times higher with mitral-valve repair (58.8\% vs. $3.8 \%)$ resulting in more heart failure-related adverse events and cardiovascular admissions [46]. When the regurgitation is only moderate, debate has centered on the role of associated MVR versus isolated coronary artery by-pass grafting (CABG) [47]. European guidelines on valvular heart disease have not clearly addressed the surgical treatment of moderate IMR and CABG. According to ESC guidelines, "there is continuing debate regarding the management of moderate ischemic MR in patients undergoing CABG and, in such cases, valve repair is preferable" [48]. Conversely, AHA/ACC guidelines consider surgical MVR at the time of CABG when the regurgitation is moderate as a class IIb-level of evidence C. This recommendation indicates that the procedure may be considered with benefit [?] risk but additional studies are needed [48]. With this level of uncertainty and patients on the operating table, a randomized clinical trial on this issue is invaluable for making decisions in these challenging patients. Recently, Michler et al. have published the 2-year outcomes of this patient population. The investigators randomized 301 patients with moderate IMR and multivessel coronary artery disease to undergo either CABG alone or CABG and MVR. The primary end point was the degree of LV reverse remodeling, as measured by means of the LV end systolic volume index (LVESVI) on transthoracic echocardiography (TTE) 1 year after randomization. All patients were followed for 2 years with end points measured at 6,12 , and 24 months. Secondary end points included findings on TTE at other time points, rate of death, MACCE, defined as a composite of death, stroke, subsequent mitral-valve surgery, hospitalization for heart failure, or worsening New York Heart Association (NYHA) class, serious adverse events, degree of postoperative mitral regurgitation, quality of life, and rehospitalization [43]. At 2 years follow up, authors concluded that the addition of mitral-valve repair to CABG had no incremental effect on reverse LV remodeling. However, patients who underwent CABG alone had 3 times higher prevalence of moderate or severe mitral regurgitation than those who underwent the combined procedure $(32.3 \%$ vs. $11.2 \%$, $\mathrm{P}<0.001)$. Conversely, this difference did not translate into higher rates of death, MACCE, serious adverse 
events (including heart failure), or readmission during these 2 years of follow up.

Moreover, patients who underwent CABG plus mitral valve repair had higher self-reported exercise capacity. Patients randomized to $\mathrm{MVR}+\mathrm{CABG}$ had longer operation time, longer cross-clamp and bypass times, which resulted in a longer postoperative length of stay during the index hospitalization, and significantly higher rates of serious neurologic events and supraventricular arrhythmias [44].

In summary, this trial concludes that patients who undergo CABG alone have less morbidity, same improvement on LV function and same rates of mortality (10.6\% vs. 10\%) and cardiovascular events than patients who undergo CABG plus MVR [44].

With these data, it would appear that the controversy is over and the problem is solved. What does IMR mean? In the current, AHA/ACC guidelines FMR occurs not only due to AMI but also reversible ischemia. If $\mathrm{MR}$ was caused by reversible ischemia rather than by nonviable scar formation, successful myocardial revascularization can lead to reduced LV size, increased mitral-valve closing forces, improved papillarymuscle synchrony, and enhanced contractility of subjacent myocardium. Therefore, treating these patients with CABG may result in a global improvement of the LV, hence improving mitral valve function.

So, one may conclude if mitral regurgitation and ventricular dysfunction may be correctable by revascularization alone, the performance of MVR would only add operative risk without any benefit.

\section{Potential Causes of Failed Surgery in Mitral Valve Repair}

There are different repair surgeries for IMR patients. Also, the mitral valve replacement has two options of mechanical and bioprosthetic. Despite failure of the two randomized controlled trials, mitral valve repair is still a viable option. The post-hoc analysis of the CTSN studies have demonstrated that in patients who had good repair with less than mild MR, the LVESVI and LV reverse remodeling was in favor of the repair patients compared to the replacement. Bouma et. al have meticulously investigated the causes of failed repair in patients undergoing IMR reparative surgery. The most sensitive and specific finding was the sphericity index of the LV with $100 \%$ sensitivity and specificity, followed by posterior tethering angle $>39.5$ degrees with $100 \%$ senility and 95\% specificity [49]. Furthermore, the basal/posterior aneurysm or dyskinesis was also associated with higher incidence of failed mitral valve repair despite a resistive mitral annular correction. Although tenting height and area have been previously recognized as important markers of failed repair in IMR, these findings were specific but not sensitive. The patient selection continues to be one of the most challenging steps to impact the outcome of these patients.

\section{Percutaneous Treatment of IMR}

MitraClip system (Abbott Inc. Irvine, CA, USA) has been approved since 2013 for the treatment of degenerative MR patients with prohibitive or high risk surgery for mitral valve repair or replacement [50]. Over $75 \%$ of patients randomized to EVEREST II trial had degenerative MR and the rest of the cohort had FMR. However, it has never been meticulously studied as to what percentage of patients randomized had IMR.

Subsequently, COAPT trial (the Cardiovascular Outcomes Assessment of the MitraClip Percutaneous Therapy for Heart Failure Patients with Functional Mitral Regurgitation) randomized 614 patients with FMR to medical therapy versus MitraClip therapy [51]. The etiology of FMR was ischemic in nearly $61 \%$ of the cohort. The subgroup analysis of the patients demonstrated that MitraClip treatment arm significantly decreased the primary endpoint of the trial (heart failure hospitalization in 2 years) as well as cardiovascular mortality and all-cause mortality. Although these results are tremendously encouraging in treating the patients with FMR due to ischemic cardiomyopathy, however, it still does not explain whether the etiology of MR in the ischemic group of MitraClip patients was IMR. In other words, the association of MR in the setting of ischemic cardiomyopathy is not consistent with the pathophsiology of IMR, as noted above. Considering all of these limitations, in our view, MitraClip therapy seems to be an excellent option for patients with IMR for the time being. Vast majority of patients with IMR already have high risk or prohibitive risk for conventional open heart surgery. Furthermore, the risk to the MitraClip procedure is extremely low, as has been proven in the COAPT trial. Therefore, a therapeutic intervention for IMR patients seems quite 
plausible. Whether treating IMR patients with MitraClip is going to change their outcomes with respect to mortality or heart failure remains to be seen. It is quite possible that we will never have another trial specifically looking into this patient population due to the heterogeneity of the disease and lack of enthusiasm from the cardiovascular community to enroll these patients into a randomized controlled trial. Furthermore, the comparator arm, either by medical therapy or surgery continues to have poor outcomes. Therefore, MitraClip therapy at the moment remains the only available percutaneous option for these patients.

\section{Conclusion}

Ischemic mitral regurgitation occurs relatively frequently in patients with ischemic heart disease and is associated with an increased risk for hospital admissions. The pathophysiology of ischemic mitral regurgitation is complex and multifactorial. The best treatment option is still debatable in majority of cases. Multidisciplinary approach by the cardiologist, heart failure specialist, Imaging specialist, structural interventional cardiologist and cardiac surgeon should be considered in all cases. The intervention should suite the patient condition and his comorbidities.

The author has no conflicts of interest to decline.

\section{Reference:}

1. Birnbaum Y, Chamoun AJ, Conti VR, et al. Mitral regurgitation following acute myocardial infarction. Coron Artery Dis 2002;13:337 - 44.

2. Grigioni F, Enriquez-Sarano M, Zehr KJ, et al. Ischemic mitral regurgitation: long-term outcome and prognostic implications with quantitative Doppler assessment. Circulation 2001;103:1759 - 64.

3. Lamas GA, Mitchell GF, Flaker GC, et al. Clinical significance of mitral regurgitation after acute myocardial infarction. Survival and Ventricular Enlargement Investigators. Circulation 1997;96:827 - 33.

4. Trichon BH, Felker GM, Shaw LK, et al. Relation of frequency and severity of mitral regurgitation to survival among patients with left ventricular systolic dysfunction and heart failure. Am J Cardiol 2003;91:538 $-43$.

5. Bouma W, van der Horst IC, Wijdh-den Hamer IJ, et al. Chronic ischaemic mitral regurgitation. Current treatment results and new mechanism-based surgical approaches. Eur J Cardiothorac Surg. 2010 Jan;37(1):170-85.

6. American Association for Thoracic Surgery Ischemic Mitral Regurgitation Consensus Guidelines Writing Committee, Kron IL, Acker MA, Adams DH, Ailawadi G, Bolling SF, Hung JW, Lim DS, LaPar DJ, Mack MJ, O'Gara PT, Parides MK, Puskas JD. 2015 The American Association for Thoracic Surgery Consensus Guidelines: Ischemic mitral valve regurgitation. J Thorac Cardiovasc Surg. 2016 Apr;151(4):940-56.

7. Borger MA, Murphy PM, Alam A, et al. Initial results of the chordal-cutting operation for ischemic mitral regurgitation. J Thorac Cardiovasc Surg. 2007 Jun;133(6):1483-92.

8. Borger MA, Alam A, Murphy PM,et al. Chronic ischemicmitral regurgitation: repair, replace or rethink? Ann Thorac Surg 2006;81:1153 - 61.

9. Otsuji Y, Handschumacher MD, Schwammenthal E, et al. Insights from three-dimensional echocardiography into the mechanism of functional mitral regurgitation: direct in vivo demonstration of altered leaflet tethering geometry. Circulation 1997;96:1999 - 2008.

10. Timek TA, Miller DC. Another multidisciplinary look at ischemic mitral regurgitation.

Semin Thorac Cardiovasc Surg. 2011 Autumn;23(3):220 - 31.

11. Magne J, Se'ne'chal M, Dumesnil JG, et al. Ischemic mitral regurgitation: a complex multifaceted disease. Cardiology 2008;112:244 - 59 . 
12. Hickey MS, Smith LR, Muhlbaier LH, et al. Current prognosis of ischemic mitral regurgitation: implications for future management. Circulation 1988;78: I51 - 9.

13. Wierup P, Nielsen SL, Egeblad H, et al. The prevalence of moderate mitral regurgitation in patients undergoing CABG. Scand Cardiovasc J 2009;43:46 - 9.

14. Kumanohoso T, Otsuji Y, Yoshifuku S, et al. Mechanism of higher incidence of ischemic mitral regurgitation in patients with inferior myocardial infarction: quantitative analysis of left ventricular and mitral valve geometry in 103 patients with prior myocardial infarction. J Thorac Cardiovasc Surg 2003;125:135 43.

15. Filsoufi F, Salzberg SP, Adams DH, et al. Current management of ischemic mitral regurgitation. Mt Sinai J Med 2005;72:105-15.

16. Birnbaum Y, Chamoun AJ, Conti VR, et al. Mitral regurgitation following acute myocardial infarction. Coron Artery Dis 2002;13:337 - 44.

17. Kaul S, Spotnitz WD, Glasheen WP, et al. Mechanism of ischemic mitral regurgitation. An experimental evaluation. Circulation. 1991 Nov;84(5):2167- 80.

18. Aguero J, Galan-Arriola C, Fernandez-Jimenez R, et al. Atrial Infarction and Ischemic Mitral Regurgitation Contribute to Post-MI Remodeling of the Left Atrium. J Am Coll Cardiol. 2017 Dec 12;70(23):2878-2889

19. Rausch MK, Tibayan FA, Miller DC, et al. Evidence of adaptive mitral leaflet growth. J Mech Behav Biomed Mater. 2012 Nov;15:208-17.

20. Quick DW, Kunzelman KS, Kneebone JM, et al. Collagen synthesis is upregulated in mitral valves subjected to altered stress. ASAIO J. 1997 May-Jun;43(3):181-6.

21. Grande-Allen KJ, Barber JE, Klatka KM, et al. Mitral valve stiffening in end-stage heart failure: evidence of an organic contribution to functional mitral regurgitation. J Thorac Cardiovasc Surg. 2005 Sep;130(3):78390 .

22. Dal-Bianco JP, Aikawa E, Bischoff J ,et al. Transatlantic Mitral Network. Myocardial Infarction Alters Adaptation of the Tethered Mitral Valve. J Am Coll Cardiol. 2016 Jan 26;67(3):275-87.

23. Kalra K, Wang Q, McIver BV, et al. Temporal changes in interpapillary muscle dynamics as an active indicator of mitral valve and left ventricular interaction in ischemic mitral regurgitation. J Am Coll Cardiol. 2014 Nov 4;64(18):1867-79.

24. Corin WJ, Monrad ES, Murakami T, et al. The relationship of afterload to ejection performance in chronic mitral regurgitation. Circulation 1987;76:59 - 67.

25. Enriquez-Sarano M, Rossi A, Seward JB, et al. Determinants of pulmonary hypertension in left ventricular dysfunction. J Am Coll Cardiol 1997;29:153 - 9.

26. Bursi F, Enriquez-Sarano M, Jacobsen SJ, et al. Mitral regurgitation after myocardial infarction: a review. Am J Med 2006;119:103 - 12.

27. Keren G, Bier A, Strom JA, et al. Dynamics of mitral regurgitation during nitroglycerin therapy: a Doppler echocardiographic study. Am Heart J 1986;112:517 - 25.

28. Hamilton MA, Stevenson LW, Child JS, et al. Sustained reduction in valvular regurgitation and atrial volumes with tailored vasodilator therapy in advanced congestive heart failure secondary to dilated (ischemic or idiopathic) cardiomyopathy. Am J Cardiol 1991;67:259 -63.

29. Rosario LB, Stevenson LW, Solomon SD, et al. The mechanism of decrease in dynamic mitral regurgitation during heart failure treatment: importance of reduction in the regurgitant orifice size. J Am Coll Cardiol 1998;32:1819 - 24 
30. Heinle SK, Tice FD, Kisslo J. Effect of dobutamine stress echocardiography on mitral regurgitation. J Am Coll Cardiol 1995;25:122 - 7.

31. St John Sutton M, Pfeffer MA, Moye L , et al. Cardiovascular death and left ventricular remodeling two years after myocardial infarction: baseline predictors and impact of long-term use of captopril: information from the Survival and Ventricular Enlargement (SAVE) trial. Circulation 1997;96:3294 - 9.

32. Greenberg B, Quinones M, Koilpillai C, et al. Effects of long-term enalapril therapy on cardiac structure and function in patients with left ventricular dysfunction. Results of the SOLVD echocardiography substudy. Circulation 1995;91:2573 - 81.

33. Konstam MA, Kronenberg MW, Rousseau MF, et al. Effects of the angiotensin converting enzyme inhibitor enalapril on the long-term progression of left ventricular dilatation in patients with asymptomatic systolic dysfunction. SOLVD (Studies of Left Ventricular Dysfunction) Investigators. Circulation 1993;88:2277 - 83.

34. Doughty RN, Whalley GA, Walsh HA, et al. CAPRICORN Echo Substudy Investigators. Effects of carvedilol on left ventricular remodeling after acute myocardial infarction: the CAPRICORN Echo Substudy. Circulation 2004;109:201 - 6 .

35. Levine RA, Schwammenthal E. Ischemic mitral regurgitation on the threshold of a solution: from paradoxes to unifying concepts. Circulation 2005;112:745 - 58.

36. Kanzaki H, Bazaz R, Schwartzman D, et al. Gorcsan 3rd J. A mechanism for immediate reduction in mitral regurgitation after cardiac resynchronization therapy: insights from mechanical activation strain mapping. J Am Coll Cardiol 2004;44:1619 - 25.

37. Sutton MG, Plappert T, Hilpisch KE, et al. Sustained reverse left ventricular structural remodeling with cardiac resynchronization at one year is a function of etiology: quantitative Doppler echocardiographic evidence from the Multicenter InSync Randomized Clinical Evaluation (MIRACLE). Circulation 2006;113:266 -72 .

38. Lancellotti P, Me'lon P, Sakalihasan N, et al. Effect of cardiac resynchronization therapy on functional mitral regurgitation in heart failure. Am J Cardiol 2004;94:1462 - 5.

39. Auricchio A, Stellbrink C, Sack S, et al, Pacing Therapies in Congestive Heart Failure (PATH-CHF) Study Group. Long term clinical effect of hemodynamically optimized cardiac resynchronization therapy in patients with heart failure and ventricular conduction delay. J Am Coll Cardiol 2002;39:2026 - 33.

40. Dı' az-Infante E, Mont L, Leal J, et al, SCARS Investigators. Predictors of lack of response to resynchronization therapy. Am J

Cardiol 2005;95:1436 - 40.

41. Fattouch K, Guccione F, Sampognaro R, et al. POINT: Efficacy of adding mitral valve restrictive annuloplasty to coronary artery bypass grafting in patients with moderate ischemic mitral valve regurgitation: a randomized trial. J Thorac Cardiovasc Surg. 2009 Aug;138(2):278-85.

42. Chan KM, Punjabi PP, Flather M, et al. Coronary artery bypass surgery with or without mitral valve annuloplasty in moderate functional ischemic mitral regurgitation: final results of the Randomized Ischemic Mitral Evaluation (RIME) trial. Circulation. 2012 Nov 20;126(21):2502-10.

43. Smith PK, Puskas JD, Ascheim DD, et al. Cardiothoracic Surgical Trials Network Investigators. Surgical treatment of moderate ischemic mitral regurgitation.N Engl J Med. 2014 Dec 4;371(23):2178-88

44. Michler RE, Smith PK, Parides MK, et al. Two-Year Outcomes of Surgical Treatment of Moderate Ischemic Mitral Regurgitation. N Engl J Med. 2016 May 19;374(20):1932-41.

45. Acker MA, Parides MK, Perrault LP, et al. Mitral-valve repair versus replacement for severe ischemic mitral regurgitation. N Engl J Med. 2014 Jan 2;370(1):23-32. 
46. Goldstein D, Moskowitz AJ, Gelijns AC, et al. Two-Year Outcomes of Surgical Treatment of Severe Ischemic Mitral Regurgitation. N Engl J Med. 2016 Jan 28;374(4):344-53.

47. Sundt TM. Surgery for ischemic mitral regurgitation. N Engl J Med 2014;371:2228-9.

48. Vahanian A, Alfieri O, Andreotti F, et al. Guidelines on the management of valvular heart disease (version 2012): the Joint Task Force on the Management of Valvular Heart Disease of the European Society of Cardiology (ESC) and the European Association for Cardio-Thoracic Surgery (EACTS). Eur J Cardiothorac Surg 2012;42: S1-44

49 Bouma W, van der Horst IC, Wijdh-den Hame, et al. Chronic ischaemic mitral regurgitation. Current treatment results and new mechanism-based surgical approaches. Eur J Cardiothorac Surg. 2010 Jan;37(1):170-85.

50. Feldman T, Foster E, Glower DD, et al. Percutaneous repair or surgery for mitral regurgitation. N Engl J Med. 2011 Apr 14;364(15):1395-406

51. Stone GW, Lindenfeld J, Abraham WT, et al. Transcatheter Mitral-Valve Repair in Patients with Heart Failure. N Engl J Med. 2018 Dec 13;379(24):2307-2318.

\section{Hosted file}

Table1 IMR.docx available at https://authorea.com/users/312423/articles/448897-ischemicmitral-regurgitation 